\title{
Plantas aquáticas em ecotecnologias: perspectivas para fitorremediação de ferro e
}

\section{manganês}

\author{
Aquatic plants in ecotechnologies: perspectives for phytoremediation of iron and manganese \\ Plantas acuáticas en ecotecnologías: perspectivas para la fitorremediación de hierro y manganeso
}

Recebido: 25/02/2021 | Revisado: 04/03/2021 | Aceito: 08/03/2021 | Publicado: 16/03/2021

\author{
Dayane Mendes Silva \\ ORCID: https://orcid.org/0000-0001-7557-8665 \\ Centro Universitário de Belo Horizonte, Brasil \\ E-mail: daybh12@gmail.com \\ Claudineia Lizieri $^{1}$ \\ ORCID: https://orcid.org/0000-0001-5704-8636 \\ Centro Universitário de Belo Horizonte, Brasil \\ Universidade Federal de Viçosa, Brasil \\ E-mail: c.lizieri@gmail.com \\ Ernandes Sobreira Oliveira Júnior \\ ORCID: https://orcid.org/0000-0002-6953-6917 \\ Universidade do Estado de Mato Grosso, Brasil \\ E-mail: ernandes.sobreira@gmail.com
}

\begin{abstract}
Resumo
Fitorremediação consiste no uso de plantas para mitigação de ambientes poluídos, tanto os terrestres como os aquáticos. Embora esta ecotecnologia tem crescido consideravelmente nas últimas décadas, a expansão de sua aplicação ainda esbarra no desafio para selecionar espécies de plantas com tal potencial. Neste trabalho, duas espécies de macrófitas aquáticas, Spirodela polyrhiza e Ricciocarpus natans, foram estudadas em experimentos laboratoriais para avaliação de seu desempenho na remoção de manganês $(\mathrm{Mn})$ e do ferro $(\mathrm{Fe})$ em solução. Plantas de $S$. polyrhiza foram testadas para ambos metais e submetidas às concentrações de 10, 15, 20, 25 e $30 \mathrm{mg} / \mathrm{L}$ de Mn e Fe. Enquanto plantas de $R$. natans foram submetidas às concentrações de 1, 2, 6 e $18 \mathrm{mg} / \mathrm{L}$ de Fe. Os resultados mostraram que plantas de $S$. polyrizha foram capazes de remover $34 \%$ do Mn e até $80 \%$ do Fe adicionados na solução. Entretanto, a redução da biomassa e do conteúdo de clorofila foi detectada nessas plantas. As plantas de $R$. natans, removeram até $50 \%$ do Fe nas concentrações de 2, 6 e $18 \mathrm{mg} / \mathrm{L}$ e não demonstraram queda da biomassa e clorofila em nenhuma das concentrações testadas, evidenciando resultados promissores para a fitorremediação de Fe. Estudos com experimentos de campo são necessários para considerar as variantes ambientais envolvidas no processo de remediação. No entanto, os achados aqui apresentados trazem, à luz da ciência, contribuições significantes para o conhecimento fitorremediador de $S$. polyrizha e $R$. natans, espécies aquáticas amplamente distribuídas nos corpos d' água brasileiros.
\end{abstract}

Palavras-chave: Macrófitas aquática; Biotecnologia; Elementos traços; Água doce.

\begin{abstract}
Phytoremediation consists of using plants to mitigate polluted environments, both terrestrial and aquatic. Although this ecotechnology has grown considerably in recent decades, the expansion of its application still faces the challenge to select plant species with such potential. In this work, two species of aquatic macrophytes, Spirodela polyrhiza and Ricciocarpus natans, were studied in laboratory experiments to evaluate its performance in removing manganese $(\mathrm{Mn})$ and iron $(\mathrm{Fe})$ in solution. Plants of $S$. polyrhiza were tested for both metals and subjected to concentrations of $10,15,20,25$ and $30 \mathrm{mg} / \mathrm{L}$ of $\mathrm{Mn}$ and Fe. While plants of $R$. natans were subjected to concentrations of 1, 2, 6 and 18 $\mathrm{mg} / \mathrm{L}$ of Fe. The results showed that plants of $S$. polyrizha were able to remove $34 \%$ of $\mathrm{Mn}$ and up to $80 \%$ of Fe added in solution. However, the reduction in biomass and chlorophyll content was detected in these plants. The $R$. natans plants removed up to $50 \%$ of Fe in concentrations of 2,6 and $18 \mathrm{mg} / \mathrm{L}$ and did not show decrease of biomass and chlorophyll in any of the tested concentrations, which show a promising result for the phytoremediation of Fe. Studies in field experiments are necessary to consider the environmental variants involved in the remediation process. However, the findings presented here bring, in the light of science, significant contributions to the phytoremediation knowledge of $S$. polyrizha and $R$. natans, aquatic species widely distributed in Brazilian water bodies.
\end{abstract}

1 autor correspondente 
Keywords: Aquatic macrophytes; Biotechnology; Trace elements; Fresh water.

\section{Resumen}

La fitorremediación consiste en utilizar plantas para mitigar ambientes contaminados, tanto terrestres como acuáticos. Si bien esta ecotecnología ha crecido considerablemente en las últimas décadas, la expansión de su aplicación aún enfrenta el desafío de seleccionar especies vegetales con tal potencial. En este trabajo, dos especies de macrófitas acuáticas, Spirodela polyrhiza y Ricciocarpus natans, fueron estudiadas en experimentos de laboratorio para evaluar su desempeño en la remoción de manganeso $(\mathrm{Mn})$ y hierro $(\mathrm{Fe})$ en solución. Las plantas de S. polyrhiza se analizaron para ambos metales y se sometieron a concentraciones de 10, 15, 20, 25 y $30 \mathrm{mg} / \mathrm{L}$ de $\mathrm{Mn}$ y Fe. Mientras que las plantas de $R$. natans se sometieron a concentraciones de 1,2, 6 y $18 \mathrm{mg} / \mathrm{L}$ de Fe. Los resultados mostraron que las plantas de S. polyrizha fueron capaces de eliminar el 34\% de Mn y hasta el $80 \%$ de Fe añadido a la solución. Sin embargo, en estas plantas se detectó la reducción del contenido de biomasa y clorofila. Las plantas de $R$. natans eliminaron hasta un 50\% de Fe en concentraciones de 2,6 y $18 \mathrm{mg} / \mathrm{L}$ y no mostraron caída de biomasa y clorofila en ninguna de las concentraciones probadas, mostrando resultados prometedores para la fitorremediación de Fe. Estudios con experimentos de campo son necesarias para considerar las variantes ambientales involucradas en el proceso de remediación. Sin embargo, los hallazgos presentados aquí aportan, a la luz de la ciencia, contribuciones significativas al conocimiento de la fitorremediación de S. polyrizha y R. natans, especies acuáticas ampliamente distribuidas en los cuerpos de agua brasileños.

Palabras clave: Macrófitos acuáticos; Biotecnologia; Oligoelementos, Agua dulce.

\section{Introdução}

Entre os principais dilemas ambientais, políticos e socioeconômicos em que a humanidade tem defrontado, a qualidade e disponibilidade da água tornaram-se um dos mais importantes, sendo relatados e discutidos há anos (Rebouças, 1999; Augusto et al., 2012; Tundisi, 2020).

Vários autores relatam que a qualidade dos ecossistemas aquáticos nas últimas décadas tem sido alterada em diferentes escalas devido à complexidade dos diversos usos da água para as atividades humanas (UNPD, 2015; Targa \& Batista, 2015, Silva et al., 2020). Este fato, consequentemente, leva à degradação ambiental e diminuição significativa da disponibilidade da água de qualidade.

Neste cenário, o setor industrial associado à demanda atual da humanidade, torna-se protagonista no processo de contaminação dos recursos hídricos (Wang et al., 2016; Singh et al., 2018), seja pela alta produção de efluentes, negligência no tratamento de seus rejeitos antes de despejá-los nos ambientes naturais e/ou acidentes ocasionados pela ausência de monitoramento. Há ainda de se considerar que o setor industrial é a principal fonte de introdução de metais pesados no ambiente aquático (Sankhla et al., 2016; Souza et al., 2016).

A contaminação de corpos d'água por metais tem sido foco de grande preocupação (Obinnaa \& Ebere, 2019; Souza et al., 2018). Os metais diferentes dos poluentes orgânicos persistem por muitos anos nos compartimentos hídricos, tais como, sedimentos de fundo e em suspensão na flora e na fauna que compõem o ecossistema hídrico (Manahan, 1999). De modo geral, eles são altamente tóxicos principalmente pela sua capacidade bioacumulativa na biodiversidade aquática (Mishra et al., 2008).

Entre os elementos traços encontrados nos corpos d'água, o ferro $(\mathrm{Fe})$ e o manganês ( $\mathrm{Mn}$ ) são frequentemente registrados. De acordo com Thornton (1995), a contaminação do corpo hídrico por Mn é proveniente, na maioria das vezes, por resíduos domésticos, atividade de mineração, processo de manufatura de metais, papéis, agentes químicos, além de depósitos de resíduos de esgotos.

O Fe é um dos elementos mais abundantes na crosta terrestre, seus compostos são encontrados em todos os corpos d'água, mesmo que em concentrações reduzidas e sua composição química é muito complexa no ambiente terrestre, sendo fortemente determinada pela diversidade de seus estados de oxidação (Russel, 1964).

Indicadores como crescimento populacional, produção em larga escala, entre outros, sugerem que a pressão sobre os recursos naturais irá aumentar, portanto, a necessidade de se desenvolver técnicas e projetos de recuperação de áreas degradadas é urgente. 
A recuperação de áreas contaminadas por metais pode ser feita através de métodos convencionais, incluindo aqueles que utilizam tratamento químico da matriz poluída (Nyer, 1998). Porém, tais procedimentos, no geral, costumam ser onerosos e com baixa sustentabilidade ecológica. Por outro lado, a fitorremediação pode ser vista como uma técnica atraente, ecologicamente viável e de menor custo, a qual já é amplamente descrita na literatura e com maior incremento em sua aplicação nos últimos anos (Raskin et al., 2000; Yan et al., 2020).

A fitorremediação consiste na utilização de espécies vegetais com potencial para remover, degradar ou isolar contaminantes orgânicos e/ou inorgânicos do meio, tanto em ambientes terrestres como os aquáticos. A técnica é uma alternativa promissora visto que tende a minimizar a destruição e desestabilização dos ecossistemas, causa baixo impacto ambiental e permite a contínua regeneração da biomassa (Cunningham, 1996 et al., USEPA et al., 2000). Esta ecotecnologia cresce anualmente nos EUA, na Europa (Pilon-Smits, 2005; Schröder et al., 2007), e mais recentemente no Brasil, sendo que as companhias de consultoria ambiental já incluem essa solução em seus pacotes de tecnologias (Mota et al., 2016).

A utilização de plantas aquáticas em processos de fitorremediação, especialmente no uso da técnica de rizofiltração, conhecida também como sistemas hidropônicos em wetlands construídos, tem obtido sucesso na mitigação de corpos d' água contaminados (Lopes \& Duarte, 2017). Este sistema tem mostrado ser viável no tratamento de esgoto doméstico e industrial, assim como em outros tipos de águas residuais, em virtude de sua natureza simples, fácil operação e rentabilidade técnicoeconômica (Brisson \& Chazarenc, 2009).

O Brasil é um país favorável à aplicação dessa ecotecnologia, pois apresenta condições climáticas propícias, alta diversidade de espécies vegetais e ambientes aquáticos amplamente poluídos. O grande desafio na aplicação da fitorremediação ainda é obter conhecimento sobre plantas tolerantes aos diversos metais presentes no meio, incluindo o Fe e Mn.

O presente trabalho analisou o potencial de duas espécies de macrófitas aquáticas (Spirodela polyrhiza e Ricciocarpus natans) para o uso em projetos ecotecnológicos de fitorremediação e biomonitoramento de águas residuais contaminadas por Fe e Mn. Para isso, o estudo buscou (i) identificar os efeitos visuais das plantas (ex. clorose, necrose, perda de folhas) sob estresse por $\mathrm{Fe}$ e $\mathrm{Mn}$; (ii) mensurar a produção de biomassa e conteúdo de clorofila sob concentrações crescentes dos elementos testados; (iii) quantificar as concentrações de metais removidas pelas plantas e (iv) determinar as concentrações tóxicas e limitantes para o processo de fitorremediação pelas espécies em estudo.

\section{Metodologia \\ 2.1 Obtenção e aclimatação das plantas}

As espécies S. polyrhiza e R. natans foram coletadas na lagoa da Universidade de Viçosa e cultivadas para obtenção de biomassa no laboratório de Fitorremediação do Centro Universitário de Belo Horizonte - UNIBH. As plantas foram acondicionadas em bandejas de polietileno contendo solução nutritiva de Hoagland (Hoagland \& Arnon, 1950), com 1/5 de força iônica, $\mathrm{pH}$ mantido entre $6,5 \pm 0,5 \mathrm{em}$ sala de crescimento de plantas, com temperatura e luz controladas $\left(23 \pm 1^{\circ} \mathrm{C}\right.$ e 230 $\mu$ mol de fótons $\mathrm{m}^{-2} \mathrm{~s}^{-1}$ ), fotoperíodo de 12 horas de luz:escuro, durante 30 dias. A solução nutritiva foi renovada a cada sete dias.

\subsection{Montagens dos experimentos}

Após obtenção da biomassa das plantas, os seguintes experimentos foram executados: (i) experimento 1 - Spirodela polyrhiza submetida às concentrações crescentes de Mn; (ii) experimento 2 - Spirodela polyrhiza submetida às concentrações crescentes de Fe e (iii) experimento 3 - Ricciocarpus natans submetida às concentrações crescentes de Fe. 
Todos os experimentos foram montados nas mesmas condições descritas no item 2.1. Para início dos experimentos, com ambas espécies, S. polyrhiza e R. natans, foram utilizadas em média 3,5 g de massa fresca (MF), acondicionados em potes de polietileno contendo $500 \mathrm{~mL}$ de solução de Hoagland. As plantas de S. polyrhiza foram submetidas às doses de 10, 15, 20, 25 e $30 \mathrm{mg} / \mathrm{L}$ de $\mathrm{Fe}$ e $\mathrm{Mn}$ nas formas de cloreto de $\mathrm{Mn}\left(\mathrm{MnCl}_{2} 4 \mathrm{H}_{2} \mathrm{O}\right)$ e fosfato de ferro $\left(\mathrm{FeHPO}_{4}\right)$. As plantas de $R$. natans foram submetidas às doses de 1, 2, 6 e $18 \mathrm{mg} / \mathrm{L}$ de $\mathrm{Fe}$ na forma de fosfato de ferro $\left(\mathrm{FeHPO}_{4}\right)$. O grupo de plantas controle foi mantido apenas em solução de Hoagland. Todos os experimentos foram conduzidos em delineamentos casualizados, com três repetições, durante sete dias e pH 6,5 $\pm 0,5$.

As concentrações utilizadas nos experimentos foram determinadas baseadas naquelas encontradas nas análises de amostra de água em áreas afetadas pelo rompimento da Barragem do Fundão (IGAM, 2015) e nas estabelecidas pela resolução do Conselho Nacional do Meio Ambiente - CONAMA 357/2005, que determina os limites de substâncias orgânicas e inorgânicas para cada tipo de classe de água.

\subsection{Sintomatologia visuais}

A observação da sintomatologia visual das plantas expostas às diferentes concentrações de $\mathrm{Mn}$ e Fe, foi realizada através do uso de microscópio estereoscópico seguida de registro fotográfico utilizando-se uma câmera de celular (IPHONE s4).

\subsection{Análise do crescimento}

No sétimo dia, a partir do início do experimento, as plantas foram removidas dos vasos, em seguida foram pesadas para obtenção da matéria fresca (MF) final e, posteriormente, colocadas em estufa convencional à $60^{\circ} \mathrm{C}$ para determinação da massa seca (MS) final. A MS inicial foi estimada a partir da média obtida de 10 repetições de plantas retiradas do mesmo lote e peso daquelas utilizadas no início dos experimentos, para cada uma das espécies. O crescimento das plantas foi expresso usando os dados de MS.

\subsection{Conteúdo de Fe e Mn remanescente na solução}

Após o término de cada experimento, subamostras da solução de cultivos das plantas foram retiradas, acondicionadas em tubos falcon, acidificadas para preservação $(\mathrm{pH} \pm 1,5)$ e, posteriormente, enviadas para quantificação da concentração de Fe e Mn remanescente na solução. As análises foram realizadas no Laboratório de Análise de Solos da Universidade Federal de Viçosa por espectrometria de absorção atômica.

\subsection{Determinação do conteúdo de pigmentos fotossintéticos}

Os conteúdos de clorofila total foram determinados a partir da extração de $0,5 \mathrm{~g}$ de MF de plantas, utilizando acetona $80 \%$ (v/v) e acrescentando-se carbonato de cálcio $\left(\mathrm{CaCO}_{3}\right)$ (Arnon,1949). A leitura da absorbância foi feita nos comprimentos de onda 646,8 nm clorofila- $b$ e 663,2 nm clorofila- $a$, em espectrofotômetro (SP-V3210 - Espectrofotômetro VIL com faixa de $325-1000 \mathrm{~nm}$ ). Os cálculos foram realizados de acordo com as fórmulas propostas por (Lichtenthaler, 1987).

\subsection{Análises estatísticas}

Os dados foram analisados estatisticamente através da análise One-Way ANOVA e Teste de Tukey $(\mathrm{p}<0,05)$ pelo software PAST de acesso livre. 


\section{Resultados e Discussão}

\section{Sintomatologia visual}

Plantas de S. polyrhiza cultivadas sob excesso de Mn em solução, a partir do quarto dia de experimento, apresentaram clorose foliar na concentração de $25 \mathrm{mg} / \mathrm{L}$. No final do experimento, plantas inteiras foram observadas em microscópio estereoscópico, sendo constatado tanto nas raízes (coifa) quanto nas frondes, manchas de coloração marrons alaranjadas, além de necroses e pontos pretos, sugerindo áreas de acúmulo de Mn (Figura 1).

Figura 1. Sintomatologia visual das plantas de Spirodela polyriza cultivadas sob excesso de Mn. A - Fronde de plantas controle; B - Fronde de plantas submetidas ao excesso de Mn, seta indicando necrose foliar; C - Detalhe da parte abaxial de planta submetida ao Mn, seta evidenciando Mn adsorvido; D - Raízes de plantas controle; E - Raízes de plantas submetidas ao Mn; F - Detalhe da raiz de plantas submetidas ao Mn, seta indicando pontos pretos na coifa sugerindo Mn acumulado. Mn = manganês.
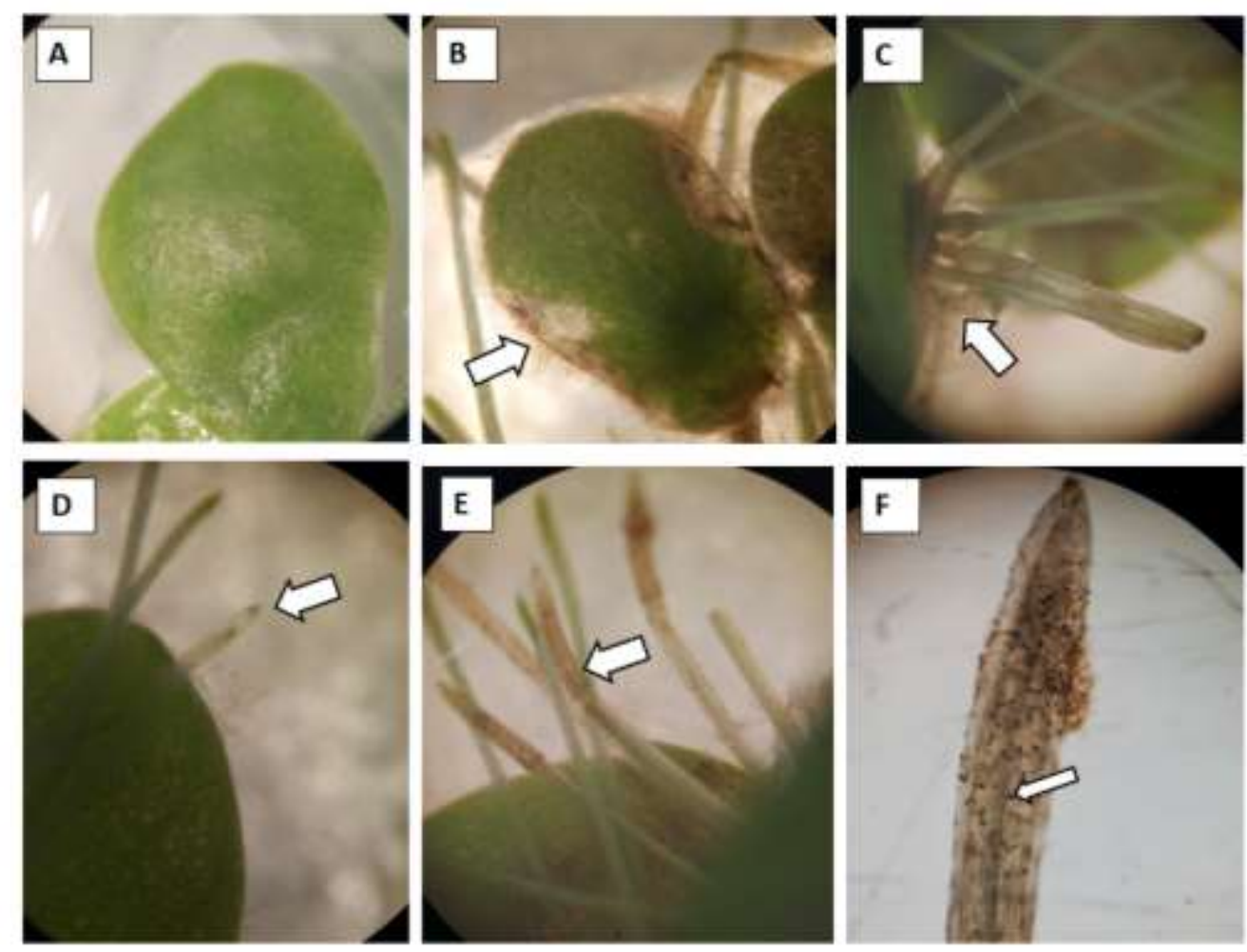

Fonte: Autores

Em plantas de S. polyrhiza tratadas com Fe os sintomas foram visíveis a partir do terceiro dia de experimento. Foi visualizado a presença de clorose e necroses em concentrações a partir de $15 \mathrm{mg} / \mathrm{L}$, tanto nas frondes como nas raízes, sendo estes efeitos mais agravados em concentrações elevadas (20 e $30 \mathrm{mg} / \mathrm{L}$ de Fe), (Figura 2). 
Figura 2. Sintomatologia visual das plantas de Spirodela polyriza cultivadas sob excesso de Fe. A - Plantas controle (esquerda) e plantas submetidas à concentração de $30 \mathrm{mg} / \mathrm{L}$ de Fe (direita); B - Frondes de plantas controle; C- Fronde de plantas submetidas à concentração de $20 \mathrm{mg} / \mathrm{L}$ de Fe; D - Fronde de plantas submetidas à concentração de $30 \mathrm{mg} / \mathrm{L}$ - seta indicando necrose foliar; E - Face abaxial de fronde de plantas submetidas ao Fe - seta indicando perda das raízes. Fe = Ferro.

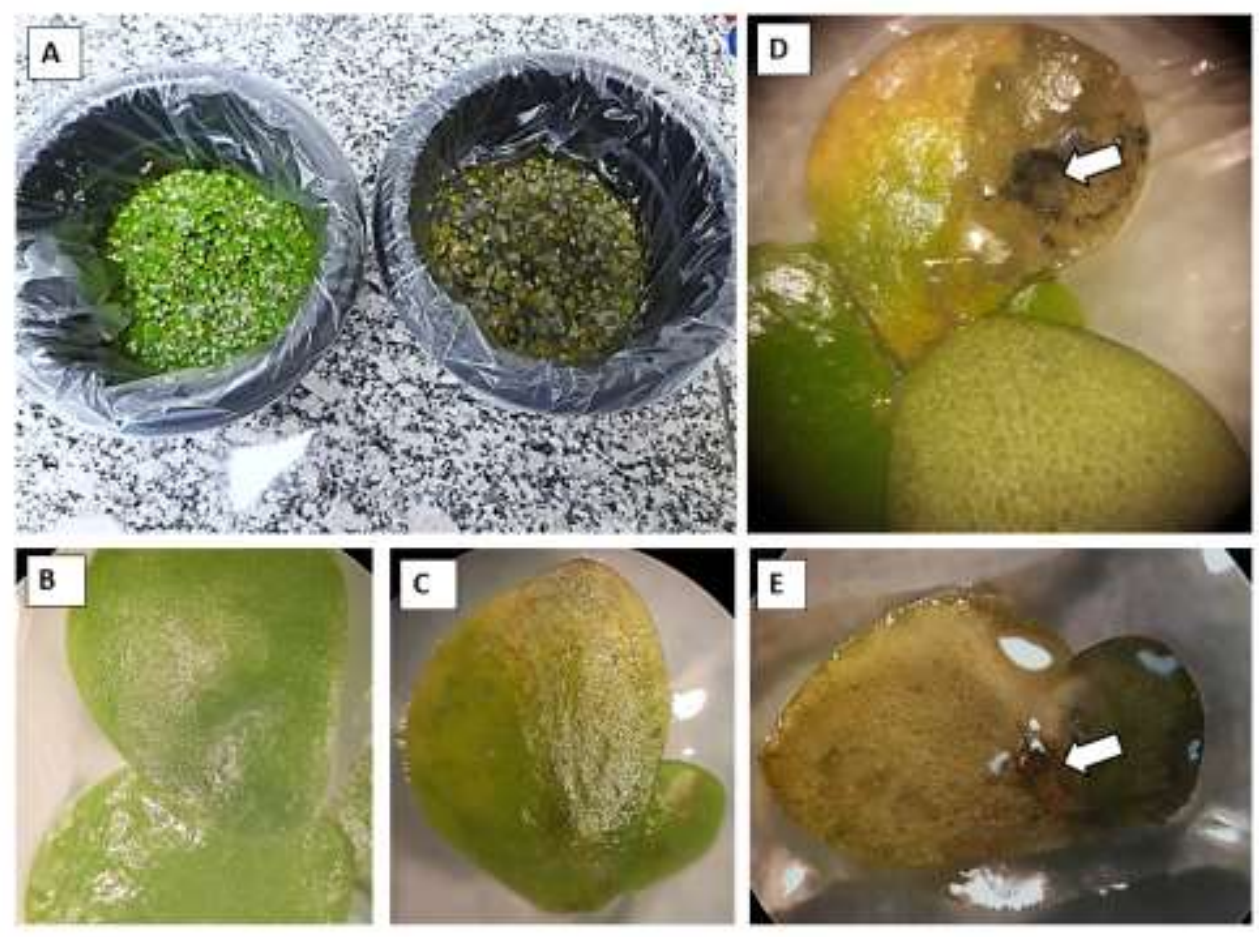

Fonte: Autores

Embora as plantas de $R$. natans tratadas com $\mathrm{Fe}$ apresentaram visualmente saudáveis quando submetidas às concentrações menores deste elemento ( 1 e $2 \mathrm{mg} / \mathrm{L}$ de $\mathrm{Fe}$ ), foi observado o crescimento de fungos na solução em todas as concentrações. A partir do quarto dia de experimento, plantas submetidas às concentrações de 25 e $30 \mathrm{mg} / \mathrm{L}$ apresentaram necrose na região adaxial (Figura 3). 
Figura 3. Sintomatologia visual das plantas de Ricciocarpus natans cultivadas sob excesso de Fe. A - Visão geral de plantas controle; B- Plantas submetidas ao excesso de Fe (20mg/L) - seta indicando o crescimento de fungos na solução; C- Detalhe da parte adaxial de planta submetida ao Fe; D - Filamentos de fungos presentes no cultivo.

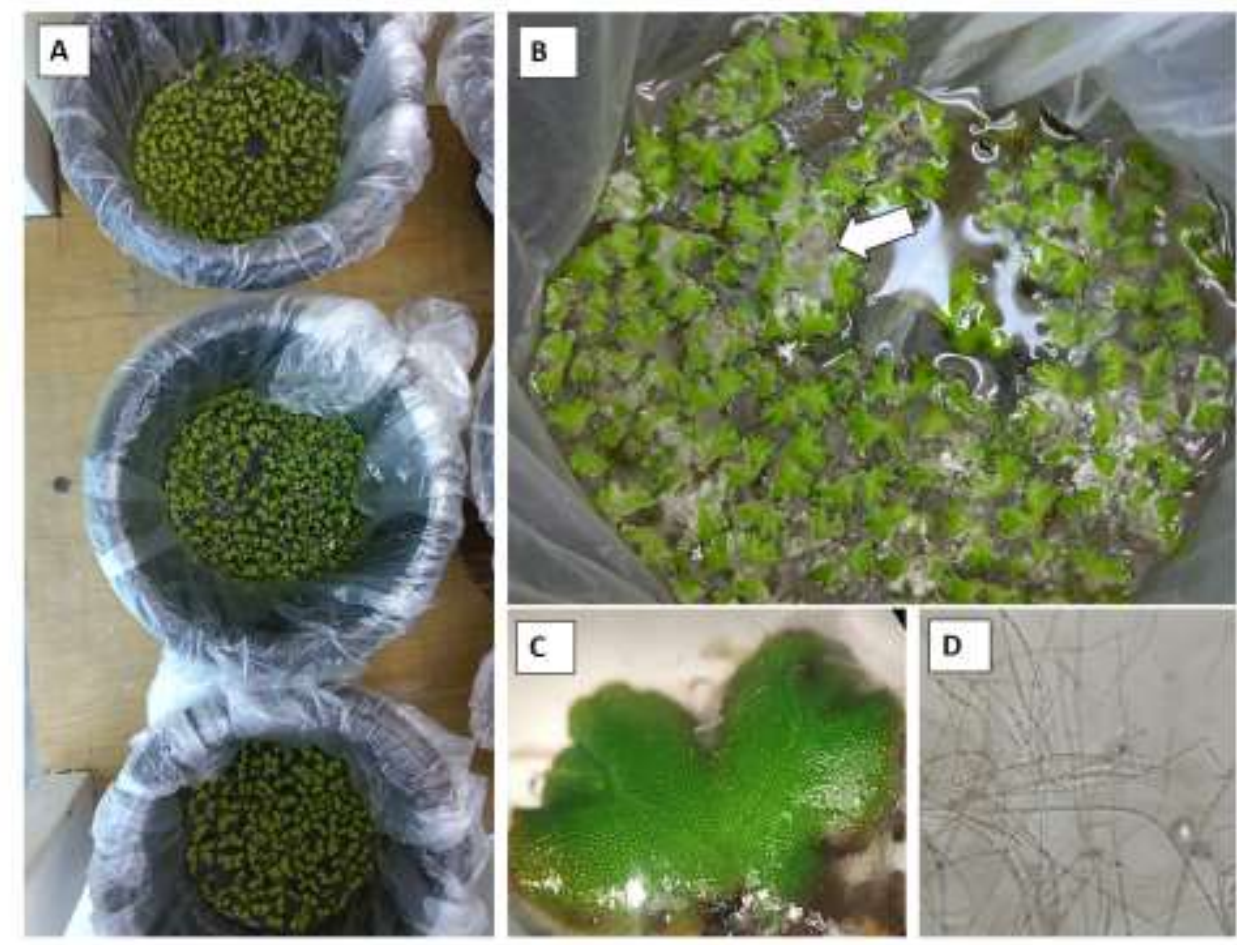

Fonte: Autores

Sintomas visuais, como clorose, têm sido relatados por Wissemeier e Horst (1992) e Kitao et al. (2001), como sendo sintomas específicos de toxidez por Mn. O surgimento de clorose nas folhas pode estar associado à redução do conteúdo de clorofila, o que foi verificado nas plantas testadas no presente estudo. Enquanto que pontos marrons têm sido indicados, em plantas terrestres, como sendo Mn oxidado (Kitao et al., 2001). Estudo prévio conduzido por Lizieri et al. (2012) também constatou alterações morfológicas e anatômicas em plantas de $S$. polyhriza tratadas com Mn, entre eles, o desarranjo do tecido aerenquimático.

A expressão dos sintomas de toxicidade de Mn depende do estado fisiológico da planta, translocação do elemento entre raízes e partes aéreas, idade foliar, bem como a distribuição de Mn e a concentração dentro célula (Kitao et al., 2001), fato evidenciado na diferenciação de sintomas de uma planta para outra ou de uma folha para outra.

S. polyhriza tratada com $\mathrm{Fe}$ apresentou necrose tanto foliar quanto no sistema radicular, principalmente nas concentrações mais elevadas, o que acarretou na diminuição da produção de biomassa. Os efeitos visuais em plantas de $R$. natans submetida ao Fe foram menos expressivos, nenhuma necrose ou clorose acentuada foram observadas nessas plantas. Informações na literatura sobre sintomas visuais do Fe em macrófitas aquáticas são escassas.

\section{Efeitos do Mn e do Fe sobre a produção de biomassa das plantas}

No final do experimento foi verificada uma tendência para perda da biomassa de plantas $S$. polyriza submetidas ao estresse por Mn em todas as concentrações, entretanto, somente plantas expostas à concentração de $30 \mathrm{mg} / \mathrm{L}$ de Mn mostraram uma redução significativa da biomassa. Essa concentração ocasionou a perda de $38 \%$ da biomassa das plantas em relação ao grupo controle (Figura 4). 
Figura 4. Biomassa de plantas Spirodela polyriza submetidas às concentrações crescentes de manganês (Mn).

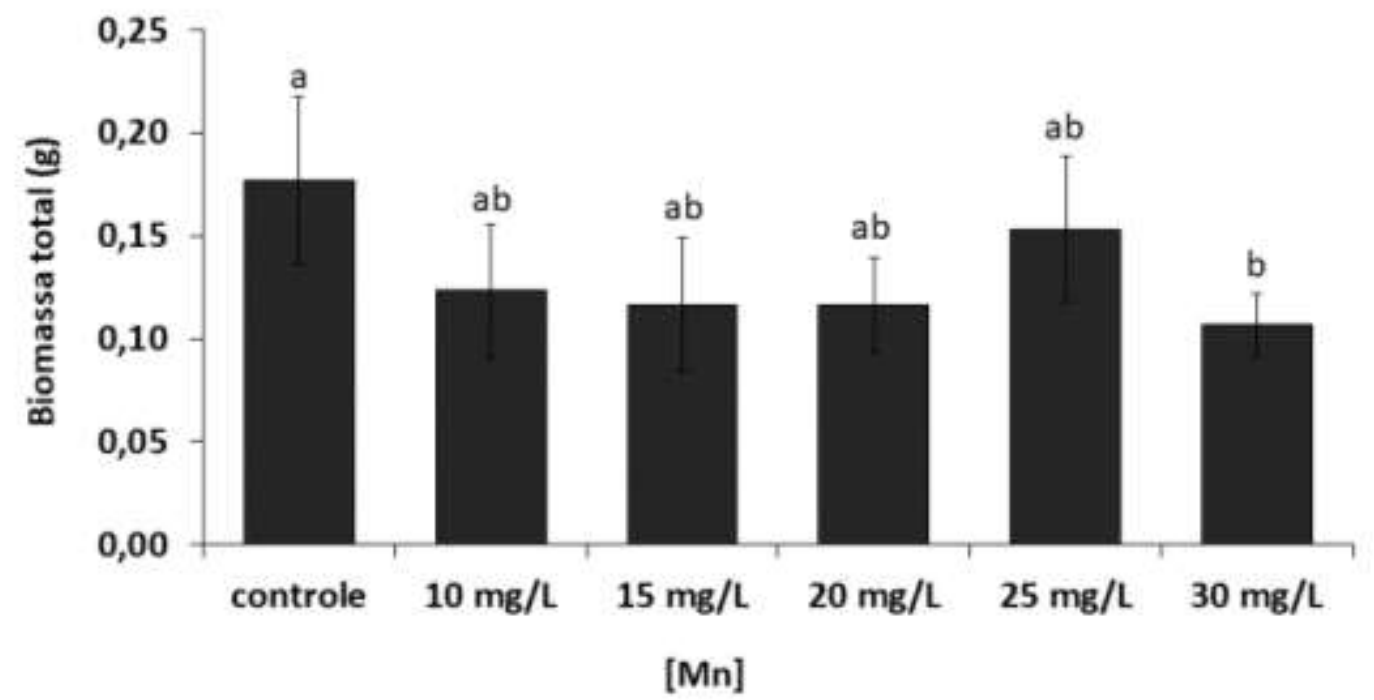

Nota: Letras diferentes sobre as barras indicam diferenças significativas $(\mathrm{p}<0,05)$. Fonte: Autores

O efeito do Fe em solução sobre a produção de biomassa de plantas S. polyrhiza foi evidentemente maior nas concentrações elevadas (Figura 5). Plantas expostas às concentrações de 20 e $30 \mathrm{mg} / \mathrm{L}$ de Fe apresentaram uma redução de $50 \%$ da biomassa total.

Figura 5. Biomassa de plantas Spirodela polyriza submetidas às concentrações crescentes de ferro (Fe).

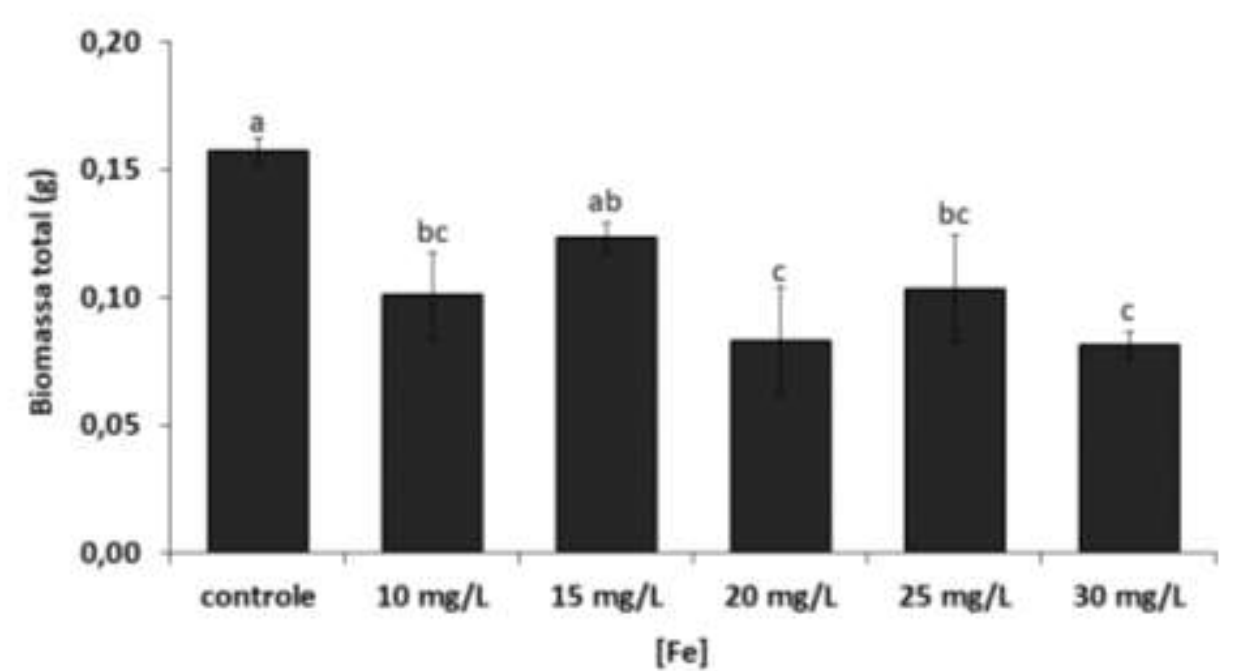

Nota: Letras diferentes sobre as barras indicam diferenças significativas $(\mathrm{p}<0,05)$. Fonte: Autores

\section{Efeitos do Fe sobre a produção de biomassa das plantas de Ricciocarpus natans}

Em plantas R. natans não foram verificadas respostas significativas para a produção da biomassa sob as diferentes concentrações de $\mathrm{Fe}$ em relação às plantas controle. Surpreendentemente, uma tendência para o aumento da biomassa foi verificada de acordo com o aumento das concentrações de Fe (Figura 6). Plantas submetidas à concentração de $18 \mathrm{mg} / \mathrm{L}$ de Fe mostram um aumento da biomassa de até $18 \%$ em relação ao grupo controle. 
Figura 6. Biomassa em plantas Ricciocarpus natans submetidas às concentrações crescentes de ferro $(\mathrm{Fe})$.

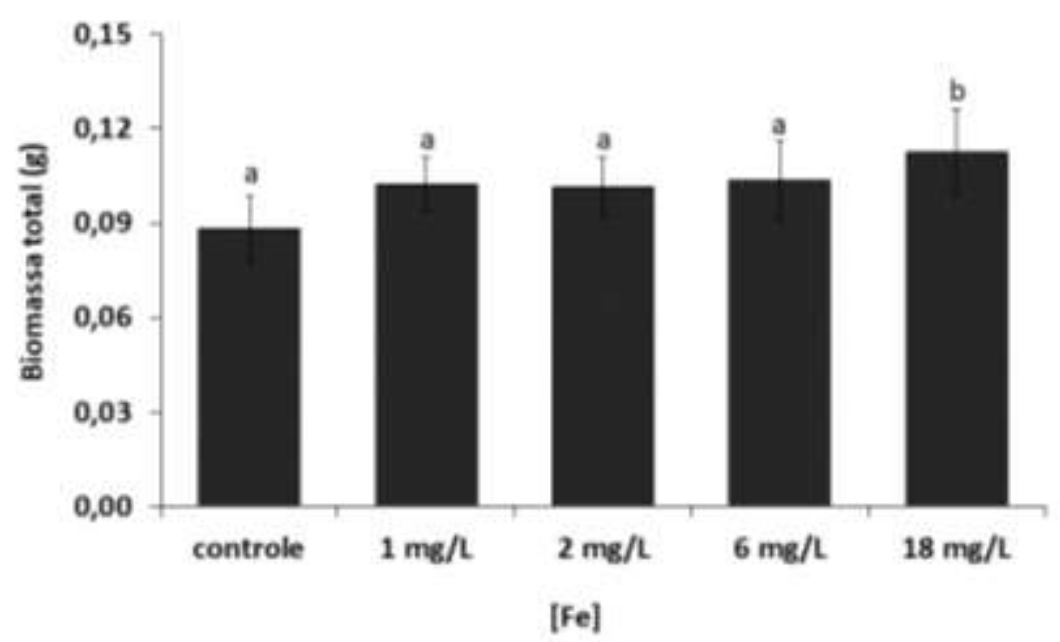

Nota: Letras diferentes sobre as barras indicam diferenças significativas $(\mathrm{p}<0,05)$. Fonte: Autores

A redução da biomassa das plantas de $S$. polyrhiza, em ambos experimentos, pode ter sido ocasionada pelo desequilíbrio nutricional causado pelo excesso do Fe e Mn na solução, adicionado ao decréscimo do conteúdo de clorofila verificado nessas plantas.

Lizieri, et. al. (2011) constataram que plantas de S. polyrhiza tratada com Mn demonstraram uma redução do crescimento a partir da concentração 0,2 mM de Mn. Sinha, et al. (1994) estudaram o efeito do Mn no crescimento de $S$. polyrhiza, em concentrações inferiores as utilizadas neste trabalho (0,01 a 0,2 $\mathrm{mM}$ de $\mathrm{Mn})$, e verificaram que a biomassa produzida por $S$. polyrhiza foi inversamente proporcional as concentrações de Mn. O presente trabalho aponta uma nova resposta para concentrações mais elevadas. As diferenças encontradas entre as espécies para o desenvolvimento de biomassa estão relacionadas às diferentes concentrações do elemento disponibilizadas na solução como também à capacidade da planta em absorver ou adsorver o metal sem afetar significativamente seus tecidos, alterando a anatomia e ativando vias de defesas contra o estresse por metal (Tangahu et al., 2011).

\section{Efeitos do Mn e do Fe sobre o conteúdo de clorofila total}

O excesso de Mn e Fe ocasionou alterações significativas nos conteúdos de clorofila total (p < 0,05) em plantas de $S$. polyrhiza. Plantas expostas às concentrações mais elevadas de Mn (15, 25 e $30 \mathrm{mg} / \mathrm{L})$ apresentaram redução de até $30 \%$ do conteúdo de clorofila total (Figura 7). Enquanto as plantas expostas às concentrações de 10 e 15 mg/L de Fe apresentaram um estímulo para biossíntese de clorofila e uma redução de até $34 \%$ deste pigmento quando expostas às concentrações de 25 e 30 mg/L, comparado aos valores obtidos para as plantas controle (Figura 8). Não foram observadas alterações significativas no conteúdo de clorofila para plantas de $R$. natans submetidas ao estresse por Fe, entretanto, plantas expostas à concentração de $18 \mathrm{mg} / \mathrm{L}$ de Fe mostraram uma tendência para aumento deste pigmento (Figura 9), sendo este resultado também observado para a produção da biomassa. 
Figura 7. Conteúdo de clorofila total em plantas de Spirodela polyrizha controle e submetidas às concentrações crescentes de manganês (Mn).

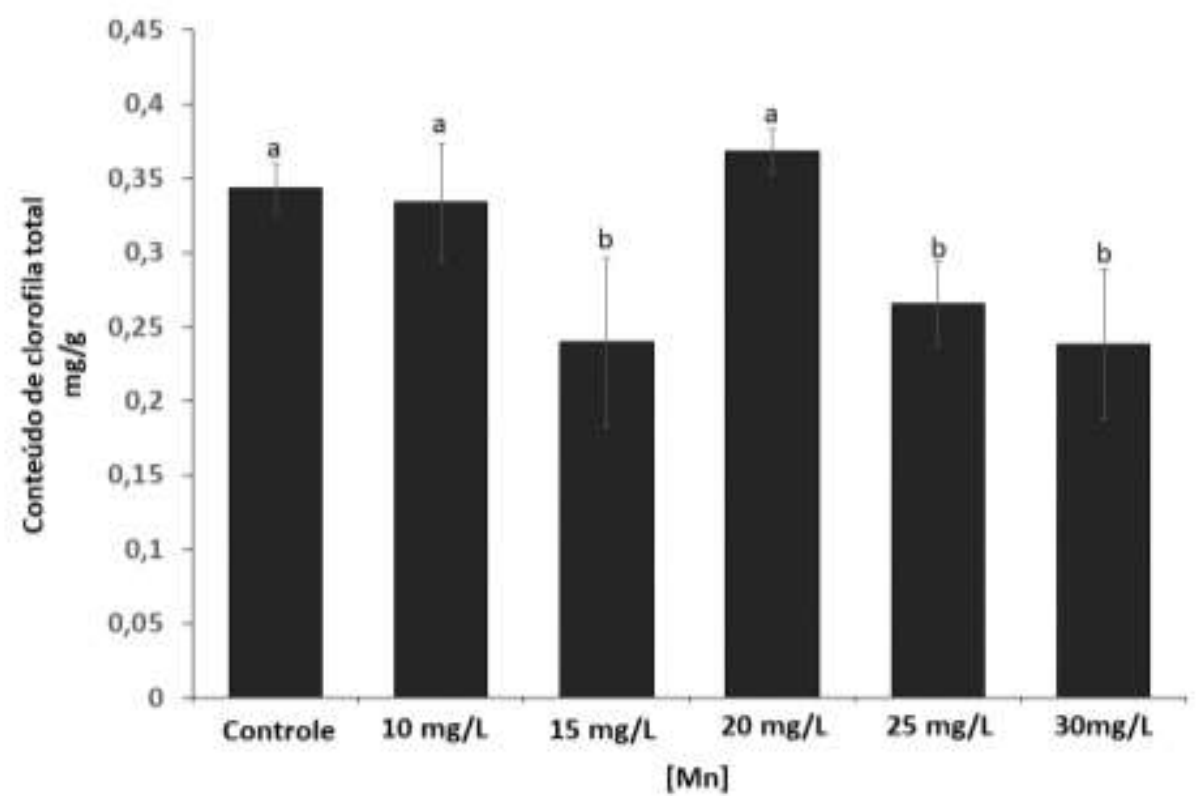

Nota: Letras diferentes sobre as barras indicam diferenças significativas $(\mathrm{p}<0,05)$. Fonte: Autores.

Figura 8. Conteúdo de clorofila total em plantas de Spirodela polyrizha controle e submetidas às concentrações crescentes de ferro $(\mathrm{Fe})$.

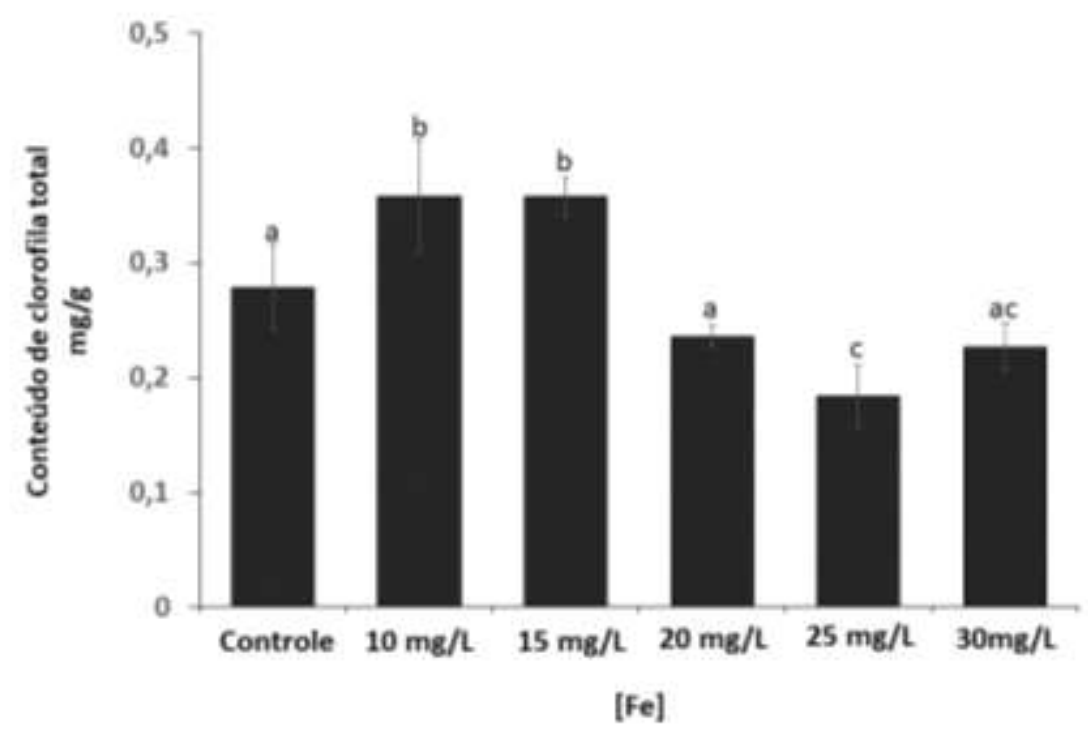

Nota: Letras diferentes sobre as barras indicam diferenças significativas $(\mathrm{p}<0,05)$. Fonte: Autores. 
Figura 9. Conteúdo de clorofila total em plantas de Riciocarpus natans controle e submetidas às concentrações crescentes de Ferro $(\mathrm{Fe})$.

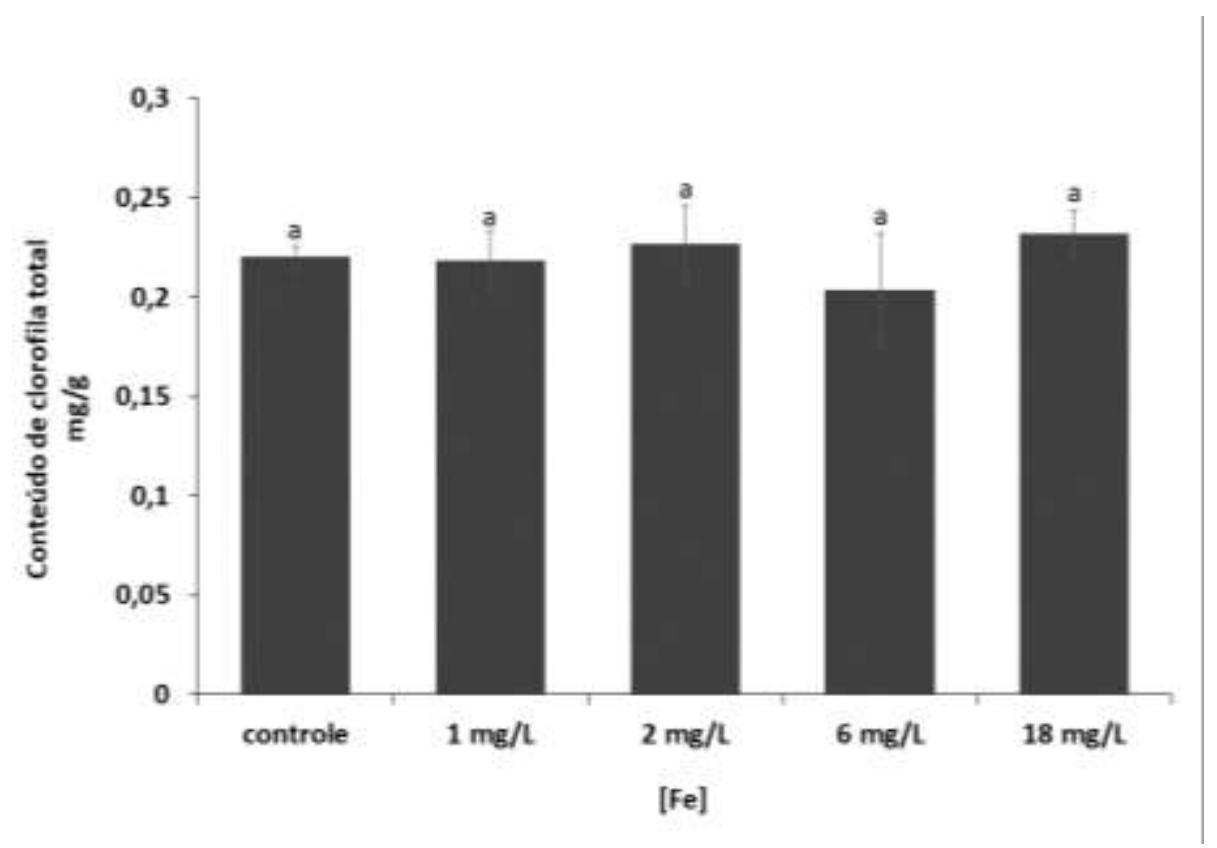

Nota: Letras diferentes sobre as barras indicam diferenças significativas $(\mathrm{p}<0,05)$. Fonte: Autores.

Diversos estudos têm demonstrado que o excesso de Mn reduz o conteúdo de clorofila (Clairmont et al., 1986; Teixeira et al., 2004, Lizieri et al., 2011). Pell et al. (1994) afirmam que toda mudança no ambiente que provoque um estresse sobre as plantas acaba provocando danos no processo fotossintético, dentre eles, a ocorrência de danos envolvendo os movimentos estomáticos, a coleta de luz e a etapa bioquímica de fixação do $\mathrm{CO}_{2}$. Estes eventos podem ocorrer envolvendo mais de um mecanismo e ser interpretado como sendo um processo tanto de atuação direta como indireta do Mn sobre a molécula de clorofila (Hauck, et al., 2002).

Nossos resultados mostraram que o $\mathrm{Fe}$ ocasionou tanto a redução como estímulo da clorofila de acordo com a concentração testada. Concentrações medianas de Fe (10 e $15 \mathrm{mg} / \mathrm{L})$ levaram aos melhores resultados de conteúdo de clorofila tanto para as plantas de $S$. polyhriza como para $R$. natans $(18 \mathrm{mg} / \mathrm{L}$ de $\mathrm{Fe}$ ), enquanto uma redução foi constatada para plantas de S. polyhriza nas concentrações mais elevadas. Solti et al. (2008) observaram alterações na atividade fotossintética induzida por $\mathrm{Fe}$, tanto para plantas como para as algas verdes. Csaturday et al. (1984) afirmam que o Fe é essencial na biossíntese da clorofila e está presente no processo de conversão da protoporfirina em protoclorofila, portanto a maior disponibilidade de $\mathrm{Fe}$ em solução poderia levar, consequentemente, ao maior biossíntese de clorofila. Enquanto Adamski (2011) observaram que plantas de batata-doce obtiveram queda na fotossíntese líquida nas concentrações de 4,5 e 9,0 mM de Fe sugerindo que estes resultados estejam diretamente ligados com a baixa formação de poder redutor $\left(\mathrm{NADPH}_{2}\right.$ e ATP) e/ou com o comprometimento de algumas reações de carboxilação. Contudo Chatterjee et al. (2006) constataram a diminuição significativa na concentração de clorofila tanto na falta quanto em excesso de Fe em Solanum tuberosum evidenciando a importância deste elemento para a biossíntese de clorofila.

\section{Fe e Mn remanescentes na solução}

As concentrações de $\mathrm{Fe}$ e $\mathrm{Mn}$ remanescentes na solução de cultivo de plantas de S. polyrhiza são apresentadas na Tabela 1. Em concentrações menores ( 10 e $15 \mathrm{mg} / \mathrm{L}$ de Fe) essas plantas foram capazes de diminuir até $84 \%$ do Fe na solução, enquanto que para o Mn, o máximo de redução foi de $34 \%$. Nota-se que os valores diminuem de acordo com o aumento das 
concentrações na solução, evidenciando uma capacidade de remoção limitada das plantas para concentrações supra ótimas desses elementos.

Tabela 1. Concentração de ferro $(\mathrm{Fe})$ e manganês $(\mathrm{Mn})$ na solução após 7 dias de exposição às plantas de Spirodella polyrizha. Valores: média da análise em triplicata *Valor do controle inicial para $\mathrm{Fe}=0,53 \mathrm{mg} / \mathrm{L} * *$ Valor do controle inicial para $\mathrm{Mn}=0,84 \mathrm{mg} / \mathrm{L}$.

\begin{tabular}{ccccc}
\cline { 2 - 5 } Concentração final de Fe e Mn (mg/L) \\
\hline Concentração inicial & \multicolumn{1}{c}{ Fe } & Redução (\%) & Mn & Redução (\%) \\
\hline Controle & $0,43^{*} \pm 0,22$ & $\mathrm{x}$ & $0,5 *^{* *} \pm 0,05$ & $\mathrm{x}$ \\
$10 \mathrm{mg} / \mathrm{L}$ & $1,74 \pm 0,09$ & 82,54 & $6,57 \pm 0,74$ & 34,3 \\
$15 \mathrm{mg} / \mathrm{L}$ & $2,26 \pm 0,13$ & 84,91 & $11,91 \pm 0,97$ & 20,6 \\
$20 \mathrm{mg} / \mathrm{L}$ & $11,83 \pm 3,36$ & 40,87 & $17,75 \pm 1,09$ & 11,22 \\
$25 \mathrm{mg} / \mathrm{L}$ & $13,7 \pm 0,69$ & 45,21 & $22,67 \pm 1,01$ & 9,3 \\
$30 \mathrm{mg} / \mathrm{L}$ & $19,33 \pm 1,34$ & 35,58 & $26,88 \pm 0,51$ & 10,38 \\
\hline
\end{tabular}

Fonte: Autores

A Tabela 2 mostra a concentração de Fe remanescente na solução de cultivo de plantas de $R$. natans. Os resultados mostraram uma redução significativa da concentração de Fe no final do experimento de mais de $50 \%$ para a maior concentração testada $(18 \mathrm{mg} / \mathrm{L})$.

Tabela 2. Conteúdo de ferro (Fe) na solução após 7 dias de exposição às plantas de Ricciocarpus natans. Valores: média da análise em triplicata. *Valor do controle inicial para $\mathrm{Fe}=0,53 \mathrm{mg} / \mathrm{L}$.

\begin{tabular}{ccc} 
& \multicolumn{2}{c}{ Concentração final (mg/L) } \\
\cline { 2 - 3 } Concentração Inicial & Fe & Redução \% \\
\hline Controle & $* 0,01 \pm 0,18$ & $\mathrm{x}$ \\
$1,0 \mathrm{mg} / \mathrm{L}$ & $0,8 \pm 0,23$ & 20 \\
$2,0 \mathrm{mg} / \mathrm{L}$ & $0,93 \pm 0,19$ & 53,5 \\
$6,0 \mathrm{mg} / \mathrm{L}$ & $2,78 \pm 0,94$ & 53,67 \\
$18 \mathrm{mg} / \mathrm{L}$ & $8,9 \pm 1,94$ & 50,56 \\
\hline
\end{tabular}

Fonte: Autores.

Há uma escassez no conhecimento sobre o mecanismo de absorção e acúmulo de Mn e Fe para outras espécies de plantas aquáticas. A literatura descreve algumas interpretações para espécies de plantas lenhosas ou arbustivas e, principalmente, para as plantas que possuem valores econômicos (Memon \& Yatazawa, 1980; Caldwell, 1998; FechtChristoffers et al., 2003; Fernando et al., 2006).

Entretanto, o potencial de S. polyrhiza em acumular diferentes contaminantes é relatado por vários autores (Tripathi \& Chandra, 1991; Sinha et al., 1994; Rai et al., 1995; Noraho \& Gaur, 1996. Lizieri et al., 2011) e o uso destas espécies em fitorremediação, vem sendo considerado promissor. Estudo prévio mostrou que S. polyrhiza absorve Fe, Mn e As (Arsênio) simultaneamente em quantidades significativas, mesmo em diferentes faixas de $\mathrm{pH}$, apresentando potencial para fitorremediação desses metais (Cruz et al., 2010). Rai et al. (1995) verificaram que S. polyrhiza foi capaz de reduzir $90 \%$ do nível de cobre de uma solução. Sinha et al. (1994) reportaram que S. polyrhiza acumulou quantidades expressivas de ferro. Nossos achados sugerem que plantas de S. polyrizha podem apresentar potencial para bioremoção de Fe e Mn em baixas 
concentrações, enquanto concentrações elevadas ocasionaram efeitos deletérios nas plantas prejudicando o processo de remoção dos elementos traços da solução.

Plantas $R$. natans também mostraram eficiência para biorremoção de Fe sem prejuízo na produção de biomassa e biossíntese de clorofila, quando cultivadas sob as concentrações de até $18 \mathrm{mg} / \mathrm{L}$, indicando alta tolerância dessa macrófita ao excesso de Fe.

A principal característica requerida para utilização de espécies vegetais na técnica de fitorremediação é o bioacúmulo do poluente associado à alta produtividade de biomassa vegetal (Mganga, 2014). No entanto, o acúmulo excessivo de metais em plantas, geralmente é conhecido por produzir respostas fisiológicas e bioquímicas danosas ao metabolismo das plantas, afetando o crescimento e desenvolvimento vegetal (Barceló \& Poschenrieder, 1990; Oliveira et al. 2001).

A sobrevivência da planta à exposição de elevados teores de metais ocorre devido à tolerância da espécie, caracterizada pela capacidade de absorver, translocar e concentrar metais em diferentes tecidos e órgãos (Marques, 1997; Mganga, 2014, Yabanli et al., 2014). A maioria das espécies vegetais tem capacidade para acumular metais, entretanto, quando elas são expostas à elevados teores, apresentam redução em seu desempenho (Larcher, 2000; Miretzky et al., 2004; Bai et al., 2018), fato que tem limitado o uso da fitorremediação para locais contaminados por altas concentrações de metais.

\section{Conclusão}

Plantas de S. polyrhiza expostas ao Fe e ao Mn sofreram efeitos tóxicos, principalmente, em concentrações elevadas, apresentando redução da biomassa e queda do conteúdo de clorofila total. Entretanto, foi constado que em ambos experimentos a espécie foi capaz de reduzir concentrações significantes dos elementos em solução.

Desde concentrações mais baixa até as mais elevadas, o Fe levou à perda de biomassa nas plantas de S. polyrhiza. Adicionado aos efeitos morfológicos deletérios, tais como, necroses acentuadas e perda das raízes, estes resultados sugerem que plantas de S. polyrhiza foram mais sensíveis ao excesso de Fe do que ao Mn.

Plantas de R. natans, mostraram boa performance nas concentrações testadas, evidenciando aumento da biomassa e do conteúdo de clorofila. Adicionalmente, essas plantas foram capazes de reduzir até $50 \%$ do conteúdo de Fe em solução, evidenciando um potencial promissor desta espécie para fitorremediação de Fe.

Estudos fisiológicos e bioquímicos, assim como pesquisa para teste em campo, são estimulados a partir deste trabalho para investigar os mecanismos que conferem às plantas de R. natans a capacidade para acumular elevadas concentrações de Fe, assim como elaboração de projetos de engenharia para aplicação dessas plantas no tratamento dos corpos d' água.

\section{Agradecimentos}

Agradecemos ao Centro Universitário de Belo Horizonte-UNIBH, a RADIX Engenharia e Software, a Fundação de Amparo à Pesquisa do Estado de Minas Gerias - FAPEMIG, pelo apoio financeiro e bolsa de Iniciação científica. Ao Prof. Eduardo Gusmão Pereira da Universidade Federal de Viçosa, campus Florestal, pelo fornecimento de reagentes laboratoriais.

\section{Referências}

Adamski, J. M. (2011). Avaliações morfofisiológicas de Ipomoea batatas L. em função da concentração de ferro. Dissertação de mestrado em Biologia. Universidade Federal de Pelotas, Pelotas.

Augusto, L.G.S, Gurgel, I. G.D., Câmara Neto, H. F., Melo, C.H. \& Costa A.M. (2012). O contexto global e nacional frente aos desafios do acesso adequado à água para consumo humano. Ciência \& Saúde Coletiva, 17(6), 1511-1522.

Arnon, D. I. (1949). Copper enzymes in isolated chloroplast polyphenol-oxidases in Betavulgaris. Plant Physiology, 24 (1), 1-15.

Bai, L., Liu, Xiao-Long \& Hu, J., Li, Ju., Wang, Zhong-Liang, Han, G., Li, Si-Liang \& Liu, Cong-Qiang. (2018). Heavy Metal Accumulation in Common Aquatic Plants in Rivers and Lakes in the Taihu Basin. International Journal of Environmental Research and Public Health, 15 (12), 2857. 
Barceló J. \& Poschenrieder C. (1990). Plant water relations as affected by heavy metal stress: A review. Journal of Plant Nutrition, 13(1), 1-37.

Brisson, J. \& Chazarenc, F. (2009). Maximizing pollutant removal in constructed wetlands: Should we pay more attention to macrophyte species selection? Science of the Total Environment. 407(13), 3923-3930.

Caldwell, C.R. (1998). Effect of elevated manganese on the ultraviolet and blue light absorbing compounds of cucumber cotyledon and leaf tissues. Journal of Plant Nutrition, 21(3), 435-445.

Chatterjee, C., Gopal, R. \& Dube, B. K. (2006). Impact of iron stress on biomass, yield,metabolism and quality of potato (Solanum tuberosum L.). Scientia Horticulturae, 108(1), 1-6.

Clairmont, K. B., Hagar, W. G. \& Davis, E. A. (1986). Manganese toxicity to chlorophyllsynthesis in Tobacco Callus. Plant Physiology, 80, $291-293$.

Cruz, M. B., Mendes P. 1, Aguiar, R., Karam D. \& Mello J W. V. (2010). IX Congreso Latino americano y del Caribe de Ingeniería Agrícola - CLIA XXXIX Congresso Brasileiro de Engenharia.

Csatorday, K., Gombos, Z. \& Szalontai, B. (1984). $\mathrm{Mn}^{2+}$ and $\mathrm{CO}^{2+}$ toxicity in chlorophyll biosynthesis. Proceedings of the National Academy of Sciences, 81, 476-478.

Cunningham, S. \& Ow, D. W. (1996). Promises and prospects of phytoremediation. Plant Physiology, 110, 715-719.

Fecht-Christoffers, M. M., Maier, P., \& Horst, W. J. (2003). Apoplastic peroxidases and ascorbate are involved in manganese toxicity and tolerance of Vigna unguiculata. Physiologia Plantarum, 177, 237-244.

Fernando, D. R., Bakkaus, E. J., Perrier, N., Baker, A. J. M., Woodrow, I. E., Batianoff, G. N., \& Collins, R. N. (2006). Manganese accumulation in the leaf mesophyll of four tree species: a PIXE/EDAX localization study. New Phytologist, 171(4), 751-758.

Hauck, M., Paul, A., Mulack, C., Fritz, E., Runge, M., 2002. Effects of manganese on the viability of vegetative diasporas of the epiphytic lichen Hypogymnia physodes. Environmental and Experimental Botany, 47(2), 127-142.

Hoagland, D. R. \& Arnon, D. I. (1950). The water-culture method for growing plants without soil. Circular. University of California Agricultural Experiment Station, 347.

IGAM - Instituto Mineiro de Gestão das Águas. (2015). Monitoramento da qualidade das águas superficiais do Rio Doce no estado de Minas Gerais. Relatório técnico.

Kitao, M., Lei, T. T., Nakamura, T., Koike, T. (2001). Manganese toxicity as indicated by visible foliar symptoms of Japanese white birch (Betula platyphylla var. japonica). Environmental Pollution, 111(1), 89-94.

Larcher, W. (2000). Ecofisiologia Vegetal. São Paulo: Pedagógica Universitária Ltda.

Lichtenthaler, H. K. (1987). Chlorophylls and carotenoids: pigments of photosynthetic biomembranes. Methods in Enzimology, 148, 350-382.

Lizieri C., Kuki K.N. \& Aguiar R. (2012). The morphophysiological responses of free-floating aquatic macrophytes to a supra-optimal supply of manganese. Water Air Soil Pollution, 223(5), 2807-20.

Lizieri, C., Aguiar, R. \& Kuki, K.N., 2011.Manganese accumulation and its effects on three tropical aquatic macrophytes: Azolla caroliniana, Salvinia minima and Spirodela polyrhiza. Rodriguésia, 62(4), 909-917.

Lopes, A. E \& Duarte, N. F. (2017). O tratamento de efluentes líquidos através de sistemas utilizando agentes de fitorremediação: uma revisão sistemática. Revista Gestão e Sustentabilidade Ambiental, 6, 432-441.

Manahan, S. E. (1999). Environmental Chemistry. (7a ed.), Lewis Publishers, Boca Raton, USA.

Marques, V. S. (1997). Efeitos de zinco e cádmio em arroz (Oryza sativa L.) cultivado em solução nutritiva e em solo tratado com lodo de esgoto enriquecido. 146 f. Dissertação Mestrado em Agronomia e Ciência do Solo. Universidade Federal Rural do Rio de Janeiro, Itaguaí. Rio de Janeiro.

Memon, A. R, \& Yatazawa, M. (1980). Distribution of manganese in leaf tissues of manganese accumulator: Acanthopanax sciadophylliodes as revealed by electronprobe X-ray microanalyzer. Journal of Plant Nutriton, 2(4): 457-476.

Mganga, N. D. (2014). The Potential of Bioaccumulation and Translocation of Heavy Metals in Plant Species Growing around the Tailing Dam in Tanzania. International Journal of Science and Technology, 3(10), 690-697.

Miretzky, P., Saralegui, A. \& Cirelli, A. F. (2004). Aquatic macrophytes potential for the simultaneous removal of heavy metals (Buenos Aires, Argentina). Chemosphere, 57(8), 997-1005.

Mishra, V. K., Upadhyaya, A. R., Pandey, S. K. \& Tripathi, B. D. (2008). Heavy metal pollution induced due to coal mining effluent on surrounding aquatic ecosystem and its management through naturally occurring aquatic macrophytes. Bioresource Technology, 99(5), 930-936.

Mota, F. A. C, \& Santana, G. P. (2016). Plantas e metais potencialmente tóxicos - estudos de fitorremediação no Brasil. Scientia Amazonia, 5, $22-36$.

Nyer, E. (1998). Groundwater and soil remediation - practical methods and strategies. Ann Arbor Press, Chelsea, Michigan.

Noraho, N. \& Gaur J. P. (1996). Cadmium adsorption and intracellular uptake by two macrophytes, Azolla pinnata and Spirodela polyrhiza. Archiv fuer Hydrobiologie, 136, 135- 144. 
Obinnaa, I. B. \& Ebere, E. C. (2019). A Review: Water pollution by heavy metal and organic pollutants: Brief review of sources, effects and progress on remediation with aquatic plants. Analytical Methods in Environmental Chemistry Journal, 2(3), 5-38.

Oliveira, J. A., Cambraia, J., Cano, M. A. O. \& Jordão, C. P. (2001). Absorção e acúmulo de cádmio e seus efeitos sobre o crescimento relativo de plantas de aguapé e de salvínia. Revista Brasileira de Fisiologia Vegetal 13(3), 329-341.

Pell, E. J., Eckart, N. \& Glick, R. E. (1994). Biochemical and molecular basis for impairment of photosynthesis potential. Photosynthesis Research, 39, 453462 .

Pilon-Smits, E. (2005). Phytoremediation. Annual Review of Plant Biology, 56, 15-39.

Rai, U. N, Sinha S., Ripathi, R. D., \& Chandra, P. (1995). Wastewater treatability Ecological Engineering, 5(1), 5-12.

potential of some aquatic macrophytes: Removal of heavy metals.

Raskin, I., \& Ensley, B. D. (2000). Phytoremediation of toxic metals: using plants to clean up the environment. John Wiley \& Sons, Inc.

Rebouças, A. C. (1999). Águas Doces no Brasil: capital ecológico, uso e conservação. Org., Rebouças, A., Braga B. \& Tundizi, J.G. São Paulo: Escrituras Editora.

Russel, J. B. (1994). Química geral. (2a ed.), Pearson Makron Books.

Schröder, P. Navarro-Aviñó, J. Azaizeh, H. et al. (2007). Using phytoremediation technologies to upgrade waste water treatment in Europe. Environmental Science and Pollution Research, 14, 490-497.

Silva, D. F. Da; Furtado, L. G.; Beltrão, N. E. S.; \& Pontes, A. N. (2020). Pressões ambientais sobre serviços ecossistêmicos hídricos em um manancial em Belém, Pará, Brasil. Research, Society and Development, 9(8), p. e502985981. Disponível em: https://rsdjournal.org/index.php/rsd/article/view/5981. Acesso em: 8 mar. 2021.

Singh, U., Singh, S., Tiwari, K. R., \& Pandey, R. S. (2018). Water Pollution due to Discharge of Industrial Effluents with special reference to Uttar Pradesh, India - A review. International Archive of Applied Sciences and Technology, 9(4), 111-121.

Sankhla, M. S., Kumari, M., Nandan, M.; Kumar, R., \& Agrawal, P. (2016). Heavy Metals Contamination in Water and their Hazardous Effect on Human Health - A Review. International Journal of Current Microbiology and Applied Sciences, 5(10), 759-766.

Sinha, S., Rai, U.N. \& Chandra, P. (1994). Accumulation and toxicity of iron manganese in Spirodela polrrhiza (L.) Schleiden. Bulletin of Environmental Contamination and Toxicology, 53, 610-617.

Souza, A. M, Salviano, A. M., Melo, J. F. B., Felix, W. P., Belém, C. S. \& Ramos, P. N. (2016). Seasonal study of concentration of heavy metals in waters from lower São Francisco River basin, Brazil. Brazilian Journal of Biology, 76(4), 967-974.

Souza, A. K. R., Morassuti, C. Y \& Deus, W. B. (2018). Poluição do ambiente por metais pesados e utilização de vegetais como bioindicadores. Acta Biomedica Brasiliensia, 9(3), 95-106.

Solti, A., Gáspár, L., Mézáros, I., Szigeti, Z., Lévai, L. \& Sárvári, E. (2008). Impact of Iron Supply on the Kinetics of Recovery of Photosynthesis in Cdstressed Poplar (Populus glauca). Annals of Botany, 102(5), 771-782.

Tangahu, B. V., Abdullah, S. R. S., Basri, H., Idris, M., Anuar, N. \& Mukhlisin, M. (2011). A Review on Heavy Metals (As, Pb, and Hg) Uptake by Plants through Phytoremediation. International Journal of Chemical Engineering, 2011, 31 pages.

Targa, M \& Batista, GT. (2015). Benefits and legacy of the water crisis in Brazil. Revista Ambiente e Água, 10(2), $234-239$.

Thornton, I. (1995). Metals in the global environment. Int. Council on Metal and the Environment.

Teixeira. I. R., Borém, A., Andrade, M. J.B., Giúdice, M. P.D. \& Cecon, R. P. (2004). Teores de clorofila em plantas de feijoeiros influenciadas pela adubação com manganês e zinco Ciências Agrárias Maringá, 26(2). 147-152, 2004.

Tripathi, R. D. \& Chandra. P. (1991). Chromium uptake by Spirodela polyrrhiza (L.) Schleiden in relation to metal chelators and pH. Bulletin of Environment Contamination Toxicology. 47 (5), 767-769.

Tundisi, J. G. \& Matsumura-Tundisi, T. (2020). A Água / José Galizia Tundisi, Takako Matsumura-Tundisi. Ed. Scienza.

UNPD - United Nations Development Programme. Beyond scarcity: power, poverty and the global water crisis. 421p.

USEPA - United States Environmental Protection Agency. (2000). Introduction to Phytoremediation.

Wang, Q. \& Yang, Z. (2016). Industrial water pollution, water environment treatment, and health risks in China. Environmental Pollution, $218,358-365$.

Yabanli, M., Yozukmaz, A. \& Sel, F. (2014). Heavy Metal Accumulation In the Leaves, Stem and Root of the Invasive Submerged Macrophyte Myriophyllum spicatum L. (Haloragaceae): An Example of Kadın Creek (Mugla, Turkey). Brazilian Archives of Biology and Technology, 57(3), 434-440.

Yan, A., Wang, Y., Tan, S. N., Yusof, M. L. M., Ghosh, S. \& Chen, Z. (2020). Phytoremediation: A Promising Approach for Revegetation of Heavy MetalPolluted Land. Frontiers in Plant Science, 11, 359. 